\title{
VERFASSUNG UND RECHT IN ÜBERSEE IN ZEITSCHRIFTEN UND JAHRBÜCHERN
}

Es fehlt der verfassungstheoretischen Erforschung der neuen Staaten stark an den erforderlichen Querverbindungen in der Arbeit und der Übersehbarkeit ihrer Ergebnisse. Der nachfolgende Bericht soll versuchen, diesem Zustand ein wenig dadurch entgegenzuwirken, daß er in Periodiken und Sammelwerken veröffentlichte Untersuchungen referiert in der Hoffnung, hierdurch eine Art von wenn auch unvollständigem Mosaik zustandebringen und Zusammenschau anzubahnen.

Begonnen sei dieser Überblick mit der Vorführung einiger Überlegungen, die Aristide R. Zolberg in einem erhellenden Aufsatz „The Structure of Political Conflict in the New States of Tropical Africa" anstellt ${ }^{1}$. Diese Arbeit ist schon deswegen wichtig, weil sie die Art und Weise überzeugend verdeutlicht, in der man die neuen Staaten Afrikas zu sehen hat, wenn man sie richtig sehen will. Hierher wiederum gehört in erster Linie eine adäquate Ansprache derjenigen Konflikte wie vor allem der Staatsstreiche, die so schockierend auf Europäer wirken. Hierzu bemerkt Zolberg grundlegend: "To understand political life in Africa, instead of viewing political disturbance as the shapeless ground surrounding institutions and processes which define the regimes of the new states, we must try to view them as characteristic processes which themselves constitute an important aspect of the regime in certain types of political systems." Mit anderen Worten: Konstituierte Institutionen und geordnete Verfahren bilden hier nicht die Regel und das Elementare nicht die regelwidrige Ausnahme; gesetztes und ursprüngliches gehören vielmehr als eine, für Europäer schwer verständliche dialektische Einheit zusammen. So kann Zolberg den IV. Abschnitt seines Aufsatzes überschreiben mit "The Coup as a Political Institution“.

Es entspricht diesem Bilde, daß Institutionen und Verfahren das Staatsgebiet nicht ausfüllen und das Staatsvolk nicht durchdringen, daß sie vielmehr lediglich eine punktuelle Existenz und Wirksamkeit zeigen. So ergibt sich auch hier statt einer Hierarchie ein schwer zu deutendes Nebeneinander: “... the new set of values, norms, and structures, which constituted an incipient national center did not necessarily grow at the expense of the older ones, as if it were a constant-sum game in which the more a country becomes 'modern' the less it remains 'traditional'." Der Verfasser schlägt daher vor, diese neuen Gebilde als "syncretic" $\mathrm{zu}$ verstehen und $\mathrm{zu}$ bezeichnen: “... since the new African states in reality do provide territorial containers for two sets of values, norms, and structures, the 'new' and the 'residual', with the latter usually subdivided into distinct sub-sets, it is useful to think of these sets as forming a particular type of u n in te g r a ted society which can be called 'syncretic'."

Dieser Dualismus zeigt sich folgerichtig in den Institutionen selbst. Wahrscheinlich urteilt man nicht zu hart, wenn man mit der Möglichkeit rechnet, sie könnten lediglich eine Fassade bilden, hinter der etwa sehr anderes lebt und wirkt. Zolberg zeigt dies im einzelnen für die politische Partei, für den Staatsdienst und "the Army', which far from being a model of hierarchical organization tends to be an assemblage of armed men who may or may not obey their officers".

Der Tenor dieses gewichtigen Aufsatzes ist nicht Skepsis, sondern ganz im Gegenteil: Er will die Sicht der Eigenart der neuen Staaten sichern, indem er inadäquate Kategorien ihrer Erfassung verbannt und auf Verständnis aus dem Wesen der Sache heraus plädiert - so fremd es auf den ersten Blick auf den Europäer wirken mag.

1 In: The American Political Science Review LXII (1968), S. $70 \mathrm{ff}$. 
Der Bericht "Politics of Manoeuvre - India in Transition", den Dilip Mukerjee in „Foreign Affairs"2 veröffentlicht, zeigt ein nicht durchweg heiteres Bild der gewürdigten Zustände und Vorgänge. Diesem Autor zufolge ist Indien zum Unterschied von anderen Entwicklungsländern belastet mit „two special problems of a politically explosive character". Das eine ist die Überproduktion, die das höhere Bildungswesen Jahr für Jahr ausstößt. Sie bewirkt eine „frustation“ der Intellektuellen, die sich wiederum in einer Neigung zu politischen Extremen Luft macht. Das zweite Übel ist eine zu schnelle Urbanisation, die einstweilen jedenfalls unlösbare Probleme aller Art schafft. Angesichts einer solchen Situation wirft Mukerjee die Frage auf, ob die gesellschaftlichen Kräfte Indiens, insbesondere seine politischen Parteien, imstande sind, diese Schwierigkeiten zu meistern.

Unter diesem Vorzeichen stimmt es den Verfasser nachdenklich, daß er keine parteipolitische Alternative zum Kongreß ${ }^{3}$ sieht. Insbesondere ist ihm zufolge die Opposition sich nur in der Negation, insbesondere in ihrer Gegnerschaft zum Kongreß einig: Positive "polarization is still not a serious possibility “. Hieraus hat sich als Folge ergeben: "The Congress has been getting flabbing for want of competition." Dieser Umstand wiederum hat das Vertrauen des Volkes untergraben "in the possibility of peaceful change via the ballot box". Es hängt somit alles $a b$ von der Leistungsfähigkeit des Kongresses. Diese sieht der Verfasser über den Mangel an Konkurrenz hinaus in mancherlei Hinsichten, und zwar nicht zuletzt aus persönlichen Gründen in Frage gestellt. Er geht den Gründen dieses Leistungsabfalles nach. Auf diese Weise gelangt er zu einem tour d'horizon aller ungelösten Probleme, die auch abgesehen von den zwei bereits erwähnten, angeblich spezifisch indischen, dieses große Land bewegen.

Es gibt schwerlich einen Gegenstand in dem Zurückhaltung in der Deutung so sehr geboten wäre wie im Falle der sogenannten chinesischen Kulturrevolution. Mit einer Mischung von Neugierde und Zweifel wendet man sich daher einem Aufsatz „The Cultural Revolution" zu, den Joan Robinson, Professor of Economics an der Universität Cambridge, von ihrem fünften Besuch in China zurückgekehrt in "International Affairs" ${ }_{4}^{4}$ veröffentlicht. Zwar beginnt sie mit der Feststellung: "It would be absurd for a foreigner to pretend fully to understand the Chinese Cultural Revolution.” Nichtsdestoweniger verschreibt sie sich der Absicht „to translate its strange terminology into familiar language". Befürchtungen, die sich angesichts einer solchen Absicht einstellen, erhalten alsbald ihre erste Bestätigung: Offensichtlich verbaut eine solche "Übersetzung" von vornherein den Zugang zu einer auch nur vielleicht richtigen Erkenntnis, wenn die Opfer dieser Revolution als "rightists“ abgestempelt werden. Vergegenwärtigt man sich ferner, daß selbst in Europa - Maurice Duverger hat hierauf die Schale seines Spottes ausgegossen "Rechts“ und "Links" alles andere als wertfreie Bezeichnungen für mögliche ideologische Positionen sind, dann hat uns die "Ubersetzung" in die Irre einer Art von moralisierender politischer Topographie geführt, die eher der Propaganda als der Erkenntnis zuzurechnen ist. So nimmt es denn auch nicht wunder, wenn man von der Verfasserin darüber belehrt wird, daß "the military-industrial complex in the United States have an interest in representing China as aggressive in order to excuse themselves...".

Unter solchen etwas beängstigenden Auspizien beginnt die Verfasserin ihre Darstellung mit einer Schilderung der Entstehung der Kulturrevolution. Die Würdigung des Gegenstandes selbst erweist sich als ergiebiger als die "leftistische" Einstellung erwarten ließ. So macht die Verfasserin etwa darauf aufmerksam, daß der Führer einer siegreichen Revolution schon um der Erhaltung seiner Stellung

2 Band 46 (1967/68), S. $519 \mathrm{ff}$.

3 Vgl. hierzu die wesentliche und konkret eindringende Studie Myron Weiners, Party Building in a New Nation - The Indian National Congress, Chicago UP 1967

4 Band 44 (1968), S. $214 \mathrm{ff}$. 
willen darauf bedacht sein müsse, ihr Feuer von Zeit zu Zeit wieder anzufachen, und zwar nicht nur gegenüber sich verbürgerlichenden Revolutionären, sondern vor allem auch gegenüber einer Jugend, die die Kampfzeit nicht mehr erlebt hat und deswegen dazu neigt, die Errungenschaften der Revolution für selbstverständlich und damit vor allem nicht für verteidigungsbedürftig zu halten.

Die letzten Seiten sind gefüllt mit einer langatmigen Rüge, die einem Autor namens Adie erteilt wird, der sich früher über das gleiche Thema geäußert hat. Begnügen wir uns daher mit der Wiedergabe eines Deutungsvorschlages, den die Verfasserin anbietet: "I offer the comment, that this (sc. die Kulturrevolution) was the first example of a new kind of class war (though inspired from above)-a revolt of the new proletariat of workers in socialist enterprises and peasants turned commune members against the incipient new class of organization men in the Communist Party."

In Band 16 (1967) der Neuen Folge des Jahrbuches des Offentlichen Rechts ${ }^{5}$ gibt der aus seiner Beschäftigung mit dem Selbstbestimmungsrecht wohlbekannte Kurt Rabl in englischer Sprache einen Überblick über das "Constitutional Development and Law of the United Republic of Tanzania“. Diese gediegene und treffsichere Arbeit bringt zunächst eine Geschichte sowohl der beiden Bestandteile dieses Gebildes wie die ihrer Vereinigung zu einem Ganzen, dessen einzigartige juristische Struktur (Bundesstaat mit nur einem Einzelstaat - es sei denn man wollte den Gesamtstaat für seinen festländischen Teil zugleich als Einzelstaat gelten lassen?; oder Einheitsstaat mit einer Region „Sansibar“? - vgl. hierzu S. 585, 604) allerdings nicht recht durchsichtig wird.

Aus der Vorführung von Verfassungsrecht und Verfassungswirklichkeit Tansanias ist vor allem die Entscheidung des Verfassunggebers für das Einparteiensystem als allgemein bemerkenswert hervorzuheben ${ }^{6}$. Historisch gesehen bedeutet dies nicht die Verdrängung anderer Parteien, da es vor Erlangung der Selbständigkeit, aber auch nachher nur die eine Partei gegeben hat, die die Freiheit erkämpfte. Die Gründe für die Konstitutionalisierung der Einzigkeit praktisch der TANU (= Tanganyika African National Union) sind nicht recht greifbar. Man kann sie wohl vor allem darauf zurückführen, daß jede weitere Partei sich mit irgendeiner Besonderheit identifizieren und damit einem „tribalism" Vorschub leisten würde, den es im Interesse der Bildung einer Nation und der Sicherung der Allgemeinheit der Staatsbürgerschaft gerade zu überwinden gilt, und dem eine einzige Partei sich eben als einzige jedenfalls dann nicht verschreiben kann, wenn sie sich entschlossen hat, Volkspartei zu sein (vgl. S. 599). Die vornehmste Wirkung dieser Lösung ist die Identität von Partei und Staat (S. 595: "State and Party are institutionally amalgated", insbesondere auch a. a. O.: "The President of the Party ... and the President of the Republic have to be one and the same person."). Angesichts dieser Konzeption versteht es sich von selbst, daß die Partei Vorrang vor dem Staat hat: Die Parteiverfassung ist deshalb Bestandteil der Staatsverfassung (S. 597). Dieser Vorrang gilt insbesondere auch für das Parlament: Nur Parteimitglieder dürfen kandidieren (S. 592); ferner legt die Partei die Politik fest, dergestalt, daß die Rolle des Parlamentes ist "reduced to auxiliary and technical functions" (S. 608; vgl. auch S. 596). Nicht zuletzt ist dieses Parlament für die Verfassungstheorie bemerkenswert, weil es sowohl (vom Präsidenten) ernannte (S. 587) wie kooptierte (S. 592) Mitglieder kennt. Da zudem für die zweite Art eine Art von präsentierenden Institutionen festgelegt ist, werden Anfänge eines ständischen Charakters dieses Parlamentes sichtbar.

Die TANU hat sich nicht für den Charakter einer Elite-, sondern den einer Massenpartei entschieden, obwohl wegen des Ausschlusses anderer Parteien die erste 
Lösung praktisch durchaus möglich gewesen wäre (vgl. S. 598). Jedermann hat freien Zutritt zur Mitgliedschaft, neuerdings wird stärker auf die Qualität der Kandidaten geachtet (S. 608). Auf der anderen Seite gibt es eine Möglichkeit politischer Betätigung nur im Schoße der einen und einzigen Partei (S. 582). Unterschiedlichkeiten der Auffassung können demgemäß nicht in Gestalt einer Auseinandersetzung zwischen mehreren Parteien, sondern müssen innerhalb der einen und einzigen Partei ausgetragen werden. Inwiefern die Eigenschaft als Volkspartei durch die 1967 angenommene Bezeichnung als Bauern- und Arbeiterpartei wieder in Frage gestellt ist, läßt sich wohl noch nicht übersehen (vgl. S. 608).

Auf dieser Grundlage hat sich die Verfassung für das Präsidialsystem entschieden (S. 590). Als Ausnahme unter den neuen Staaten Afrikas hat Tansania auf Grundrechte verzichtet: Nach dem Vorbild Englands soll es der Richter sein, der die Freiheit gegen das Unrecht zu verteidigen hat (S. 601); er kann sich hierbei auf die Präambel der Verfassung stützen (S. 602), die nicht etwa "unverbindlicher Gesetzesinhalt" ist, sondern Rechtssätze höchsten Ranges enthält.

Vor allem für die Wirtschaftsverfassung sind die "Arusha Resolutions" der TANU von Januar 1967 erheblich. Sie verlangen „effective control over the principal means of production" (S. 606), obwohl es an anderer Stelle wieder heißt, Tansania sei „a state of Peasants and Workers, but is not yet a Socialist State“. Die Nationalisierung, die gegen "full and fair compensation“ erfolgen soll, hat bisher ausländische und indische Unternehmen erfaßt (S. 607).

Der zweite Band des Jahrbuches „Verfassung und Verfassungswirklichkeit" ${ }^{\text {"7 bringt }}$ u. a. einen Bericht aus der Feder Axel Ridders über "Wahlen und außerparlamentarische Machtbildung in Indonesien". Diese Arbeit hat sich zum Ziel gesetzt die Prüfung der Frage, ob und wieweit die Übernahme des niederländischen Vielparteiensystems zum Scheitern des ersten demokratischen Versuchs in diesem Lande beigetragen hat. Alle seit 1945 gegebenen Verfassungen haben dem Autor zufolge "die elementarsten Spielregeln einer Demokratie mißachtet: Die Notwendigkeit der Schaffung systemkonformer Institutionen". Hiermit ist angesprochen das Unterbleiben der Stiftung neuer "pouvoirs intermédiaires“, insbesondere integrations- und aktionsfähiger politischer Parteien. Die Statistiken, die der Verfasser heranzieht, zeigen in der Tat ein erschreckendes Bild hoffnungsloser Zersplitterung. Verantwortlich für diese Zersplitterung und hierdurch bedingte Labilität der Regierungen macht der Verfasser nicht zuletzt die Entscheidung für die Verhältniswahl. Hierdurch „war das Vielparteiensystem fest im Bewußtsein der Parteien und der Offentlichkeit zementiert. Nun konnte eine Entwicklung anheben, wie sie in Europa so oft vorexerziert worden ist: Schwierigkeit der Regierungsbildung, instabile Koalitionsregierungen, Ideologisierung der Parteien aus einem Gegegenseitig-sich-der-Schuld-Bezichtigen der Koalitionsparteien in Krisensituationen, Machtlosigkeit des Premierministers, Abwertung des Parlamentes in der Offentlichkeit, Anwachsen der radikalen und extremen Parteien von links und rechts, Ruf nach dem starken Mann, Aufkommen unkontrollierbarer außerdemokratischer Machtgruppen und letzten Endes die Diktatur.“ (S. 268/269).

HERBERT KRÜGER 